\title{
Malnutrition Incidence Among Inpatients: A Cross-Sectional Retrospective Study
}

\author{
Serap ANDAÇ ÖZTÜRK ${ }^{1 *}$, Nihal Altun², Gökçen Garipoğlu ${ }^{3}$ \\ 1. Health Science Faculty, Istanbul Aydın University, Inonu Street 38, Istanbul, 34295, Turkey \\ 2. Department of Nutrition, Sultan II. Abdülhamid Han Education and Research Hospital, Istanbul, 34668, \\ Turkey \\ 3. Health Science Faculty, Bahçeşehir University, Istanbul, 34349, Turkey \\ *E-mail of the corresponding author: serapozturk@aydin.edu.tr
}

\begin{abstract}
Malnutrition in hospital is a significant problem which involves clinical complication risks, lengthened hospital stay and worsening prognosis. In order to overcome this problem, it is important to identify malnutrition in patients. The aim of this study was to retrospectively research the malnutrition risk of patients at time of admission and to create awareness about the importance of malnutrition screening. This research is a cross-sectional descriptive retrospective study which screened inpatients in İstanbul Sultan Abdülhamid Han Education and Research hospital from August 2018 to January 2019. Research data and NRS-2002 scores were retrospectively gathered from patient files. Between the dates of the study, a total of 10,060 patients stayed in the hospital and of these $490(4.9 \%)$ were identified to be malnourished. Of these patients, $0.87 \%$ developed malnutrition after admission. The clinic with highest malnutrition was the anesthesia intensive care (25.9\%). There was a significant increase identified in NRS2002 scores with age $(\mathrm{p}=0.001)$; as NRS-2002 scores increased the mortality rates were found to significantly increase $(\mathrm{p}=0.015)$. The mortality in the patient group with NRS- 2002 scores of 7 was $50 \%$. Malnutrition screening will contribute to monitoring malnourished patients from time of admission and reducing mortality rates. According to the results of our study, the malnutrition risk is higher among elderly patients and as NRS-2002 score increases mortality increases. Due to this correlation between inpatient NRS-2002 scores and mortality, it was concluded that rapid screening and close surveillance of nutritional interventions for patients with high scores are important in terms of mortality..
\end{abstract}

Keywords: malnutrition, NRS, length of stay, nutrition

DOI: $10.7176 / \mathrm{JHMN} / 76-10$

Publication date:June 30th 2020

\section{Introduction}

Malnutrition may be defined as insufficiencies in energy, proteins and other nutrients (Bolayir et al., 2019) and is frequently seen among hospitalized patients (Javid Mishamandani et al., 2019), though the prevalence changes according to the society and definitions used (Bavelaar et al., 2008). Hospital malnutrition is a public health problem which increases risk of clinical complications such as length of hospitalization, worsens prognosis, increases mortality and spending related to health-rehabilitation (Bavelaar et al., 2008; Kyle, Kossovsky, Karsegard \& Pichard, 2006; Raupp et al., 2018) and delays wound healing (Bolayir et al., 2019). According to study results from a variety of countries in Europe, malnutrition rates are from $13-75 \%$ and nearly $50 \%$ is not diagnosed (Bolayir et al., 2019).

Assessment of nutrition is important for identification of patients at risk in terms of nutrition and to plan interventions. However, as this is in-depth research including medical history, physical examination, biochemical parameters, anthropometric measurements, body composition and assessment of nutrient intake, it is timeconsuming and is not easy to apply to every hospitalized patient (Bolayir et al., 2019). Though there are many screening (NRS-2002) and assessment (SGA, MNA) methods for assessment of nutritional status, there is no gold standard (Budzyński, Tojek, Czerniak \& Banaszkiewicz, 2016). Experts agree that patients requiring nutritional support should receive this support in the shortest time possible during their hospital stay. Early intervention increases the success of nutritional treatment and prevents worsening of nutritional status (Eglseer, Halfens \& Lohrmann, 2017), reduces hospital costs, shortens length of stay and reduces readmission requirements (Budzyński, Tojek, Czerniak \& Banaszkiewicz, 2016). As a result, screening is an important stage in good nutritional care. With this aim, valid screening tools should be used; however, hospitals generally do not apply this screening and most patients do not receive diagnosis or treatment (Eglseer, Halfens \& Lohrmann, 2017). Many screening tools have been developed in the name of screening inpatients in terms of nutritional risks. All have strong and weak 
aspects; however, the European Society for Clinical Nutrition and Metabolism (ESPEN) recommends the use of NRS-2002 for inpatients (Rabito et al.; Hersberger et al., 2019). The NRS-2002 creates a risk score linked to malnutrition risk related to diseases, changes in nutritional status, severity of disease and patient age and reduction in patient body mass index and was shown to be beneficial for identification of malnutrition risk (Knappe-Drzikova et al., 2019; Avelino-Silva \& Jaluul, 2017).

The aim of this study was to retrospectively research the malnutrition risk of inpatients at time of admission to hospital. Additionally, this study will create awareness about the importance of malnutrition screening in order to contribute to improving nutritional status, shortening length of stay and reducing complication development.

\section{Material\&Metods}

\subsection{Research Method}

This research was a cross-sectional, descriptive, retrospective study, with necessary institutional permission for the study obtained from the Provincial Health Directorate (Number: 16867222-604.01.01).

\subsection{Research Population and Data Collection}

The research population comprised inpatients in İstanbul Sultan II. Abdülhamid Han Education and Research Hospital with patients admitted for treatment between August 2018 and January 2019 retrospectively screened. Research data and NRS-2002 scores were retrospectively collected from patient files.

\subsection{Analysis of Data}

Analysis of data used the SPSS 21 program. Fit to normal distribution of data was assessed with the Shapiro Wilks test. Descriptive statistics calculated mean, standard deviation, number and percentage distribution. Analysis of grouped data used the chi square test, while analysis of continuous variables used the T test.).

\section{Results}

A total of 10,060 patients were admitted from 1 August 2018 to 31 January 2019 to the research population of İstanbul Sultan Abdülhamid Han Education and Research Hospital. Of these patients, 490 (4.9\%) were identified to be malnourished (NRS-2002 $\geq 3$ ) and were monitored by the nutrition team. While 87 malnourished patients $(0.87 \%)$ were not identified to have malnutrition at time of admission, malnutrition developed during the hospital stay of these patients (identified on $2^{\text {nd }}$ or later screenings).

Of the 490 patients monitored by the nutrition team, 242 were female and 248 were male. The mean NRS-2002 scores for females and males were $3.6 \pm 0.7$ and $3.6 \pm 0.9$ according to gender and this was not statistically significant $(P=0.745)$. The age interval for patients was $18-100$ years, with mean of $73.1 \pm 15.8$ years. The distribution of patients according to NRS-2002 scores are presented in Table 1.

Table 1: Distribution of patients according to NRS-2002 scoring

\begin{tabular}{lrr}
\hline NRS-2002 Score & $\mathrm{n}$ & $\%$ \\
\hline 3 & 269 & 54.9 \\
4 & 169 & 34.5 \\
5 & 35 & 7.1 \\
6 & 13 & 2.7 \\
7 & 4 & 0.8 \\
\hline Total & 490 & 100.0 \\
\hline
\end{tabular}

The distribution of patients monitored by the nutrition team for malnutrition diagnosis is shown in Table 2 . The anesthesia intensive care was the clinic with most malnutrition identified at $25.9 \%$, while the dermatology and underwater medicine clinics had lowest malnutrition identified at $0.2 \%$. 
Table 2: Distribution of patients with malnutrition according to clinic

\begin{tabular}{|c|c|c|}
\hline CLINIC & $\mathrm{n}$ & $\%$ \\
\hline Anesthesia Intensive Care & 127 & 25.9 \\
\hline Internal Medicine Intensive Care & 49 & 10.0 \\
\hline Brain surgery Intensive Care & 9 & 1.8 \\
\hline Dermatology & 1 & .2 \\
\hline Brain surgery ward & 4 & .8 \\
\hline Internal Medicine ward & 51 & 10.4 \\
\hline Endocrinology & 2 & .4 \\
\hline Infectious diseases ward & 6 & 1.2 \\
\hline Gastroenterology ward & 12 & 2.4 \\
\hline General Surgery ICU* & 5 & 1.0 \\
\hline General Surgery ward & 5 & 1.0 \\
\hline Chest Diseases ward & 76 & 15.5 \\
\hline Chest diseases ICU & 19 & 3.9 \\
\hline Cardiology ICU & 11 & 2.2 \\
\hline Nephrology ward & 4 & .8 \\
\hline Neurology ward & 37 & 7.6 \\
\hline Neurology ICU & 46 & 9.4 \\
\hline Palliative care ward & 15 & 3.1 \\
\hline CVD ward & 1 & .2 \\
\hline ENT $\dagger$ & 3 & .6 \\
\hline CVD ICU & 1 & .2 \\
\hline Urology ward & 3 & .6 \\
\hline Hematology ward & 2 & .4 \\
\hline Underwater medicine & 1 & .2 \\
\hline Total & 490 & 100.0 \\
\hline
\end{tabular}

* ICU; Intensive Care Unit $\dagger$ ENT; Ear Nose Throat

When NRS-2002 scores are compared according to age groups, there was a significant increase identified between NRS-2002 scores with age $(* P=0.001$, Table 3$)$.

Table 3: Distribution of age groups according to NRS-2002 scoring

\begin{tabular}{lrcrc} 
& \multicolumn{5}{c}{ NRS-2002 Score } \\
\cline { 2 - 5 } Age (years) & \multicolumn{1}{c}{$\mathrm{n}$} & $\overline{\mathrm{x}}$ & $\mathrm{sd}$ & \multicolumn{1}{c}{$P$} \\
\hline $18-30$ & 9 & 3.7 & 1.1 & \\
$31-60$ & 86 & 3.3 & .6 & $* P=0.001$ \\
$>61$ & 395 & 3.7 & .8 & \\
\hline Total & 490 & 3.6 & .8 & \\
\hline
\end{tabular}

The mean hospital stay of patients with malnutrition diagnosis was $54.7 \pm 5.1$ days. When duration of stay is investigated according to NRS-2002 scores, those with NRS-2002 score of 3 had mean duration of stay of 57.8 days, while for those with score of 6 it was 72.69 days and for those with score of 7 it was 19.9 days. This situation was not statistically significant (data not shown) $(\mathrm{p}=0.732)$.

When the discharge status of patients according to NRS-2002 scores is investigated, 312 were discharged home, 144 died and 34 were transferred to another hospital. As the NRS-2002 scores of patients increased, the mortality rates were found to significantly increase $(* P=0.015)$ (Table 4$)$. 
Table 4: Patient discharge status according to NRS-2002 scoring

\begin{tabular}{lcccc}
\hline \multirow{2}{*}{$\begin{array}{l}\text { NRS-2002 } \\
\text { Score }\end{array}$} & \multicolumn{4}{c}{ Discharge status } \\
& Ex & $\begin{array}{c}\text { Discharge } \\
\text { home }\end{array}$ & Transfer & P \\
\hline 3.00 & 67 & 186 & 16 & \\
4.00 & 54 & 101 & 14 & $* P=0.015$ \\
5.00 & 15 & 19 & 1 & \\
6.00 & 6 & 4 & 3 & \\
7.00 & 2 & 2 & 0 & \\
\hline Total & 144 & 311 & 31 & \\
\hline
\end{tabular}

When feeding routes are examined according to NRS-2002 scores of patients, the most commonly chosen routes were oral feeding and total parenteral feeding (TPN) (Table 5).

Table 5: Distribution of patients with NRS-2002 score of 3 or more according to feeding route

\begin{tabular}{lrr}
\hline Feeding route & $\mathrm{n}$ & \multicolumn{1}{c}{$\%$} \\
\hline Enteral & 98 & 20.0 \\
Oral + Enteral & 4 & 0.8 \\
TPN* & 109 & 22.2 \\
TPN + Enteral & 11 & 2.2 \\
Oral & 191 & 39.0 \\
TPN + Oral & 20 & 4.1 \\
Not feeding & 57 & 11.6 \\
\hline Total & 490 & 100.0 \\
\hline
\end{tabular}

*TPN: Total Parenteral Nutrition

\section{Discussion}

This research was performed with the aim of retrospectively determining the incidence of malnutrition among inpatients treated in İstanbul Sultan Abdülhamid Han Education and Research Hospital. From 1 August 2018 to 31 January 2019, a total of 10,060 patients were admitted and patient files were assessed in terms of malnutrition scores and it was identified that 490 patients were monitored by the nutrition team due to malnutrition (Table 1). Of these patients, 242 were female and 248 were male. Screening between these dates determined the incidence of malnutrition was $4.9 \%$. According to NRS-2002 score at time of admission, $4.9 \%$ of patients were malnourished, while $0.87 \%$ of patients developed malnutrition during hospital stay when assessed with repeat screening. In the literature, the malnutrition incidence varies from 20-50.2\% (Rabito et al., 2017; Knappe-Drzikova et al., 2019; Correia, Perman \& Waitzberg, 2017) and some patients were malnourished at admission, while the majority developed malnutrition later (Rabito et al., 2017). Similar to our study, another study assessing patient malnutrition status with NRS-2002 found this rate was 6.4\% (Budzyński, Tojek, Czerniak \& Banaszkiewicz, 2016). Additionally, generally the mean rate for malnutrition among inpatients is given as $41.7 \%$ (Tobert, Mott \& Nepple, 2018). In the present study the lower prevalence of malnutrition may be due to the study including all branches in the hospital with a high variety of patients (from branches with low malnutrition risk to intensive care admissions), while differences compared to studies in the literature may be due to differences in methods used for malnutrition screening (use of tools like SGA, MUST, NUTRIC).

When the incidence of malnutrition according to clinic is examined, the clinic with most malnutrition was the anesthesia intensive care at $25.9 \%$, the chest diseases clinic at $15.5 \%$, the internal medicine clinic at $10.5 \%$ and the internal medicine intensive care clinic at 10\%. Additionally, the clinics with lowest malnutrition incidence were dermatology and underwater medicine at $0.2 \%$. Considering the chronic disease load of patients admitted to the anesthesia and internal medicine clinics, the high incidence of malnutrition is expected. Additionally, when low malnutrition rates are assessed, the patient profile in the dermatology and underwater medicine clinics is expected to have low malnutrition, confirming this expectation. However, contrary to expected, palliative care was found to have very low values for malnutrition rates (Table 2).

Malnutrition in intensive care is a serious problem around the world, with prevalence reported as $50.8-78.1 \%$ in developed and developing countries, respectively (Burgos, Joaquín, Blay \& Vaqué, 2019), and this rate identified as $24.5 \%$ among individuals with chronic obstructive disease (Marco et al., 2019). Studies in Asia have shown rates of $87 \%$ in critical patients and $56 \%$ in surgical patients (Correia, Perman \& Waitzberg, 2017). Though there were low rates in our study compared to other studies, when the distribution according to clinic is investigated, 
clinics with high malnutrition expected in terms of patient profile were observed to have high rates. Malnutrition affects all patient groups, while the elderly are found to be more sensitive and elderly individuals with malnutrition at time of admission stay in hospital for longer durations and have higher mortality (Avelino-Silva \& Jaluul, 2017). Again, similar to the literature, when NRS-2002 scores are compared according to age groups, there was a significant increase in NRS-2002 scores identified with age $(* P=0.001$, Table 3$)$. A similar study assessing malnutrition with NRS-2002 reported malnourished patients were older (Budzyński, Tojek, Czerniak \& Banaszkiewicz, 2016).

The mean duration of hospital stay among patients with malnutrition diagnosis was $54.7 \pm 5.1$ days. When NRS2002 scores are investigated in terms of duration of stay, the mean stay was 57.87 days for those with NRS-2002 score of 3, and 19.9 days for those with score of 7, with no statistical significance found (data not shown, $\mathrm{p}=0.732$ ). Contrary to the literature, in spite of the increase in NRS-2002 score, the reason for the shortened duration of stay may be due to the mortality in this group being 50\%. When the discharge status of patients is investigated according to NRS-2002 scores, 312 were discharged home, 144 died and 34 were transferred to another hospital. As the NRS-2002 score of patients increased, the mortality rate was found to significantly increase $(* P=0.015)$ (Table 4). It is emphasized that for inpatients with malnutrition, high morbidity and mortality may be prevented by taking care during nutritional care of these patients (Hersberger et al., 2019). A study reported the mortality of malnourished patients increased 3.3 times compared to patients without nutritional problems (odds ratio) (Pasquini, Neder, Araújo-Junqueira \& De-Souza, 2012).

When the feeding route of patients is examined according to NRS-2002 scores, the most commonly chosen route was oral feeding (39\%) followed by total parenteral feeding $(22 \%)$ (Table 5). A study with malnutrition incidence of $50.2 \%$ found that in spite of the high prevalence, less than $9 \%$ of patients received enteral or parenteral nutrition (Correia, Perman \& Waitzberg, 2017).

In the literature it is generally stated that patients do not receive diagnosis or treatment for malnutrition (Eglseer, Halfens \& Lohrmann, 2017), while a study in Brazil reported $61 \%$ of malnourished patients received nutritional treatment (Pasquini, Neder, Araújo-Junqueira \& De-Souza, 2012). Again, it is possible to encounter various rates from 27.9-23.2\% to 50\% in the literature (Khalatbari-Soltani \& Marques-Vidal, 2016). Variations in these figures may be due to various criteria being used in studies like only oral nutrition solution or enteral feeding.

Our study identified the rate of malnutrition diagnosis and feeding intervention (oral nutrition solution, enteral feeding and parenteral applications) as 59.3\% and assessed the malnutrition monitoring and surveillance of inpatients as satisfactory.

\section{Conclusion}

Due to the possible outcomes of malnutrition, the use of a valid scale for malnutrition screening at time of admission to hospital is important in terms of planning nutritional support. Monitoring patients from time of admission will contribute to reducing morbidity and mortality rates. According to the results of our study, older patients have greater risk of malnutrition and as NRS-2002 score increases mortality increases. Contrary to the literature, the rate of patients identified as having malnutrition during repeated malnutrition screening during the hospital stay was low. Due to the correlation between NRS-2002 score of inpatients with mortality, it was concluded that rapid screening and close monitoring of nutritional interventions of patients with high score is important in terms of mortality.

\section{References}

Avelino-Silva, T. J., Jaluul, O. (2017). Malnutrition in hospitalized older patients: Management strategies to improve patient care and clinical outcomes. International Journal of Gerontology, 11(2), 56-61.

Bavelaar, J. W, Otter, C. D., van Bodegraven, A. A., Thijs, A., van Bokhorst-de van der Schueren, M.A.E. (2008). Diagnosis and treatment of (disease-related) in-hospital malnutrition: The performance of medical and nursing staff. Clinical Nutrition, 27(3), 431-438.

Bolayir, B., Arik, G., Yeşil, Y., Kuyumcu, M. E.,, Varan H. D., Kara, Ö., Güngör, AE., Balam Yavuz, B., Cankurtaran, M., Gülhan, M., Halil, M. G. (2019). Validation of Nutritional Risk Screening-2002 in a hospitalized adult population. Nutrition in Clinical Practice, 34(2), 297-303.

Budzyński, J., Tojek, K., Czerniak, B., Banaszkiewicz, Z. (2016). Scores of nutritional risk and parameters of nutritional status assessment as predictors of in-hospital mortality and readmissions in the general hospital population. Clinical Nutrition, 35(6), 1464-1471.

Burgos, R., Joaquín, C., Blay, C., Vaqué, C. (2019). Disease-related malnutrition in hospitalized chronic patients with complex needs. Clinical Nutrition, In Press https://doi.org/10.1016/j.clnu.2019.06.006

Correia, M. I. T. D., Perman, M. I., Waitzberg, D. L. (2017). Hospital malnutrition in Latin America: A systematic 
review. Clinical Nutrition, 36(4), 958-967.

Eglseer, D., Halfens, R. J. G., Lohrmann, C. (2017). Is the presence of a validated malnutrition screening tool associated with better nutritional care in hospitalized patients? Nutrition, 37, 104-111.

Hersberger, L., Bargetzi, L., Bargetzi, A., Tribolet, P., Fehr, R., Baechli, V., Geiser, M., Deiss, M., Gomes, F., Kutz, A., Kägi-Braun, N., Hoess, C., Pavlicek, V., Schmid, S., Bilz, S., Sigrist, S., Brändle, M., Benz, C., Henzen, C., Nigg, M., Thomann, R., Brand, C., Rutishauser, J., Aujesky, D., Rodondi, N., Donzé, J., Stanga, Z., Mueller, B., Schuetz, P. (2019). Nutritional risk screening (NRS 2002) is a strong and modifiable predictor risk score for short-term and long-term clinical outcomes: secondary analysis of a prospective randomised trial. Clinical Nutrition, In Press https://doi.org/10.1016/j.clnu.2019.11.041

Javid Mishamandani, Z., Norouzy, A., Hashemian, S. M., Khoundabi, B., Rezaeisadrabadi, M., Safarian, M., Nematy, M., Pournik, O., Jamialahmadi, T., Shadnoush, M., Moghaddam, O. M., Zand, F., Beigmohammadi, M. T., Khoshfetrat, M., Shafiei, E., Sedaghat, A. (2019). Nutritional status of patients hospitalized in the intensive care unit: A comprehensive report from Iranian hospitals, 2018. Journal of Critical Care, 54, 151158.

Khalatbari-Soltani, S., Marques-Vidal, P. (2016). Impact of nutritional risk screening in hospitalized patients on management, outcome and costs: A retrospective study. Clinical Nutrition, 35(6), 1340-1346.

Knappe-Drzikova, B., Maasberg, S., Vonderbeck, D., Krafft, T. A., Knüppel, S., Sturm, A., Müller-Nordhorn, J., Wiedenmann, B., Pape, U. F. (2019). Malnutrition predicts long-term survival in hospitalized patients with gastroenterological and hepatological diseases. Clinical Nutrition ESPEN, 30, 26-34.

Kyle, U. G., Kossovsky, M. P., Karsegard, V. L., Pichard, C. (2006). Comparison of tools for nutritional assessment and screening at hospital admission: A population study. Clinical Nutrition, 25(3), 409-417.

Marco, E., Sánchez-Rodríguez, D., Dávalos-Yerovi, V. N., Duran, X., Pascual, E. M., Muniesa, J. M., Rodríguez, D. A., Aguilera-Zubizarreta, A., Escalada, F., Duarte, E. (2019). Malnutrition according to ESPEN consensus predicts hospitalizations and long-term mortality in rehabilitation patients with stable chronic obstructive pulmonary disease. Clinical Nutrition, 38(5), 2180-2186.

Pasquini, T. A. S., Neder, H. D., Araújo-Junqueira, L., De-Souza, D. A. (2012). Clinical outcome of protein-energy malnourished patients in a Brazilian university hospital. Brazilian Journal of Medical and Biological Research, 45(12),1301-1307.

Rabito, E. I., Marcadenti, A., Da Silva Fink, J., Figueira, L., Silva, F. M. (2017). Nutritional Risk Screening 2002, Short Nutritional Assessment Questionnaire, Malnutrition Screening Tool, and Malnutrition Universal Screening Tool are good predictors of nutrition risk in an emergency service. Nutrition in Clinical Practice, 32(4), 526-532.

Raupp, D., Silva, F. M., Marcadenti, A., Rabito, E. I., da Silva Fink, J., Becher, P., Gottschall, C. (2018). Nutrition screening in public hospital emergency rooms: Malnutrition Universal Screening Tool and Nutritional Risk Screening-2002 can be applied. Public Health, 65, 6-8.

Tobert, C. M., Mott, S. L., Nepple, K. G. (2018). Malnutrition diagnosis during adult inpatient hospitalizations: Analysis of a multi-institutional collaborative database of academic medical centers. Journal of the Academy of Nutrition and Dietetics, 118(1), 125-131. 